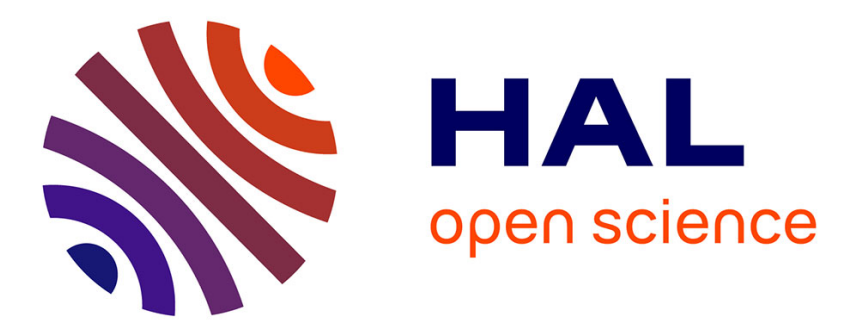

\title{
MONOTONICITY PROPERTIES FOR MULTISERVER QUEUES WITH RENEGING AND FINITE WAITING LINES
}

Oualid Jouini, Yves Dallery

\section{- To cite this version:}

Oualid Jouini, Yves Dallery. MONOTONICITY PROPERTIES FOR MULTISERVER QUEUES WITH RENEGING AND FINITE WAITING LINES. Probability in the Engineering and Informational Sciences, 2007, 21, pp.335-360. 10.1017/S0269964807000010 . hal-01264901

\section{HAL Id: hal-01264901 \\ https://hal.science/hal-01264901}

Submitted on 29 Jan 2016

HAL is a multi-disciplinary open access archive for the deposit and dissemination of scientific research documents, whether they are published or not. The documents may come from teaching and research institutions in France or abroad, or from public or private research centers.
L'archive ouverte pluridisciplinaire HAL, est destinée au dépôt et à la diffusion de documents scientifiques de niveau recherche, publiés ou non, émanant des établissements d'enseignement et de recherche français ou étrangers, des laboratoires publics ou privés. 


\title{
Monotonicity Properties for Multiserver Queues with Reneging and Finite Waiting Lines
}

\author{
Oualid Jouini \& Yves Dallery \\ Laboratoire Génie Industriel, Ecole Centrale Paris \\ Grande Voie des Vignes, 92295 Châtenay-Malabry Cedex, France. \\ walid.jouini@ecp.fr, yves.dallery@ecp.fr
}

Probability in the Engineering and Informational Sciences, 21:335-360, 2007

\begin{abstract}
We consider a markovian multiserver queue with a finite waiting line in which a customer may decide to leave and give up service if its waiting time in queue exceeds its random deadline. We focus on the performance measure in terms of the probability of being served under both transient and stationary regimes. We investigate monotonicity properties of first and second order of this performance with respect to the buffer size, say $k$. Under the stationary regime, we prove that our service level is strictly increasing and concave in $k$, whereas we prove under the transient regime that it is only increasing in $k$.
\end{abstract}

Keywords multiserver queues; blocking; reneging; performance measures; convexity properties; coupling arguments.

\section{Introduction}

Monotonicity properties of performance measures are useful for understanding and solving optimization problems of queueing systems. Optimization models are being used increasingly in the design of a variety of systems where queueing phenomena arise. Examples include flexible manufacturing systems, as well as service systems and telecommunications networks. For such problems, it is important to know the convexity properties of the performance measures with respect to the design variables. These properties may enable us to reduce the performance optimization problem to a convex programming problem which is easier to solve. Using a convexity result, Yao and Shanthikumar [34] accelerate their computation procedure to design a loss queueing system subject to constraints on the loss probability. Koole and Pot [19] consider an optimization problem for an $M / M / s / K+M$ queue. The objective function is a profit function of the number of servers and the buffer size. They derive some monotonicity properties about the defined performance measure. Based on these properties, they develop a fast algorithm which avoids the research of all possible solutions to get the global optimum.

Several convexity properties about various performance measures have been investigated in 
the queueing literature. The major performance measures for delay systems are the average waiting time, the average queue length and the probability of delay. Those for pure loss systems include basically the probability for a new arrival to be lost. In general, the loss probability is related to systems involving finite buffers or systems with reneging. In this paper, we consider a queueing system with impatient customers and finite waiting line. The performance measure of interest is the probability for a new arrival customer to enter service. Or equivalently, the probability to not be lost. We investigate first and second order monotonicity properties of our performance measure as a function of the queue size. Note that the design of the buffer size is an important issue in practice. Koole et al. [18] address this problem by investigating the maximum queue length during a busy period for an infinite buffer size.

Another central feature in many practical queueing systems is the reneging phenomenon, i.e., one customer may decide to leave the queue (abandons) before starting service. For instance, call abandonment is not negligible in call centers operations. A major drawback in many call center models is assuming customers to be infinitely patient. Garnett et al. [7] show using numerical examples that models with and without abandonment tend to give very different performance measures even if the abandonment rate is small. A further application would be manufacturing systems with perishable items. In this paper, we analyze the simplest abandonment model, assuming that the customers patience is exponentially distributed. However, the model is still of interest in practice as mentioned by Pierson and Whitt [25]. The authors have shown, using various simulation experiments, that the $M / M / s+M$ model provides a good approximation of the $M / G I / s+G I$ model.

Here is how the rest of the paper is organized. In Section 2, we review the literature close to our work. In Section 3, we present the framework of the work: Section 3.1 is devoted to formulate the queueing model, and Section 3.2 gives definitions and some preliminary results. In Section 4, we focus on the first order monotonicity results. In Section 4.1, we start by proving two helpful lemmae before proceeding to the main result. Next, we establish using coupling arguments that the transient and stationary probabilities of being served are increasing in the buffer size. In Section 4.2, we prove the result for the stationary performance measure using an analytical approach. In Section 5, we prove that the stationary probability of being served is strictly concave in the buffer size. Some numerical illustrations of the results are also presented. In Section 6, we conclude and propose some directions for future research. 


\section{Literature Review}

In this section, we review the literature related to this paper. We start by presenting some papers investigating monotonicity results for models without reneging. Second, we focus on those for models incorporating reneging.

We classify the results for models with infinitely patient customers into three classes: pure loss, limited buffer and infinite buffer models. For pure loss systems, Harel [9] proves that the throughput of an $M / G / s / s$ is concave in the arrival and service rates. He also characterizes the traffic intensity below which the Erlang loss formula is convex in the arrival rate, and above which it is concave. Furthermore, he shows that the Erlang loss formula is convex in the service rate. For the same model, Messerli [22] proves that the loss probability is a convex function of the number of servers. Additional properties of the loss probability are also discussed by Jagerman [13].

As for systems with limited buffer, Nagarajan and Towsley [23] investigate the convexity of the loss probability in the $M / M / 1 / K$ queue with respect to the traffic intensity and the service rate. They show that the loss probability is convex in the service rate. However, they prove that there is a value of the traffic intensity which exactly delineates the convex and concave regions of the loss probability as a function of the traffic intensity. Pacheco [24] considers for his part a more general model with many servers, namely the $M / M / s / K$ queue. He proves that the loss probability is convex in the queue capacity. Meister and Shanthikumar [21] prove many convexity results for tandem queueing systems. Several interesting stochastic comparisons of various variants of multiserver queues with limited buffer are also derived by Berger and Whitt [4].

In what follows, we review some monotonicity results for models with infinite queue capacity. Tu and Kumin [28] prove that the expected number of customers in a $G / G / 1$ queue is convex in the service rate. They also show that the result does not hold for a $G I / G I / 2$ queue. Surprisingly, Harel [10] show that the expected number of customers in an $M / D / s$ queue is convex in both arrival and service rates. For the $M / M / s$ queue, Lee and Cohen [20] show that the average queue length and the probability of delay, are both convex in the arrival rate. For the same model, Harel and Zipkin [12] establish that the average sojourn time, as well as its standard deviation are convex in arrival and service rates. Again about the $M / M / s$ queue, Jagers and van Doorn [14] focus on the performance measure in terms of the probability for a 
customer to wait no longer than a given threshold. Note that this service level is widely used in call centers. The authors show that the probability of interest is concave as a function of the number of servers, if the latter is strictly greater than the offered load. We refer the reader for further convexity properties to Weber [30] and [31], Grassmann [8], Shanthikumar [27], Harel and Zipkin [11], Shaked and Shanthikumar [26] and Koole [17].

Let us now turn to the second area of literature related to this paper. Queues with impatient customers have received some attention in the queueing literature. The results focus especially on performance evaluation. We refer the reader to Ancker and Gafarian [1], Garnett et al. [7], and references therein for simple models assuming exponential reneging times. Other papers have allowed reneging to follow a general distribution. Related studies include those by Baccelli and Hebuterne [3], Brandt and Brandt [6], Ward and Glynn [29], and Zeltyn and Mandelbaum [35]. Concerning the monotonicity properties, few results were derived. This is due to the mathematical complexities of such problems. Bhattacharya and Ephremides [5] consider multiserver queues with impatient customers. They show that the transient number of lost customers is a monotone function with respect to the arrival rate, the service rate, as well as the reneging rate. Armony et al. [2] consider a holding cost in an $M / M / C$ queue with impatient customers. They prove that this function is decreasing and convex in the service rate and the number of customers. Some sensitivity results for the Erlang- $A$ model can also be found in Whitt [32].

\section{Framework}

This section is devoted to formulate the general framework of the paper. First, we describe the queueing system and detail the processes assumptions. Second, we define the performance measures of interest, namely, the fraction of customers who get service under both transient and stationary regimes. We next develop some preliminary results.

\subsection{Model Formulation}

Consider a multiserver queueing system with a single class of customers. The model consists of a set of $s$ parallel, identical servers and a finite queue (waiting line). There is a maximum number of customers that may be simultaneously present, we assume that the system can hold at most a total of $K$ customers including those in service. Clearly $K \geq s$, and we denote the queue capacity by $k=K-s, k \geq 0$. The system is operated in such a way that at any time, 
any customer can be addressed by any server. So upon arrival, a customer is addressed by one of the available servers, if any. If not, the customer joins the queue if less than $K$ customers are present in system. If not, the customer is refused entry and departs immediately without service. He is blocked and considered lost. In addition, we assume that customers are impatient. After entering the queue, a customer will wait a random length of time for service to begin. If service has not begun by this time, he will renege (abandon), and again considered to be lost. Finally, retrials are ignored, and reneging is not allowed once a customer starts his service.

The arrival of customers is assumed to follow a Poisson process. Interarrival times are i.i.d. and exponentially distributed with rate $\lambda$. Successive service times are assumed to be i.i.d., independent from the arrival process, and follow an exponential distribution with rate $\mu$. Times before reneging are assumed to be i.i.d., and exponentially distributed with rate $\gamma$. Following similar arguments, the system can be modeled as an $M / M / s / K+M$ queue. The symbol $M$ after the + is to indicate the markovian assumption for reneging times. Note that owing to reneging, the system is always ergodic even if the queue has infinite capacity. Also, ergodicity would always be assured for our system because of its limited capacity, even if the customers were assumed to be infinitely patient. In conclusion, the system we consider here is unconditionally ergodic.

As we shall establish later, we need not to specify here the scheduling policy, except the assumption that it is workconserving. A scheduling policy is defined to be workconserving (nonidling) if the waiting customers get into service as soon as a server is free. No server stays idle as long as there are customers to serve. Several familiar disciplines of service as the first come, first served (FCFS), the last come, first served (LCFS), the random selection (RS) disciplines are characterized to be workconserving. A further possible classification for scheduling policies is the preemption/non-preemption schemes. In preemptive cases, we may interrupt the service of a customer to let a new arrival start service. However, a discipline of service is said to be non-preemptive if there is no possibility for service interruption.

\subsection{Preliminaries}

In this section, we focus on characterizing the performance measure of interest. It is defined in terms of the fraction of customers who get service, i.e., the fraction of customers who are not blocked and who do not renege. 
Let us consider an interval of time $[0, t], t>0$. We initially assume that the system starts empty. Given that $t$ units of time have elapsed, let $n(t)$, and $s(t)$ be the total number of arrivals (including blocked customers), and the number of those who enter service, respectively. We define the transient fraction of customers who enter service, $Q(t)$, during $[0, t]$ as $Q(t)=\frac{s(t)}{n(t)}$. Taking the limit as $t \rightarrow \infty$ of $Q(t)$ leads to the stationary fraction $Q$ of successful departures, $Q=\lim _{t \rightarrow \infty} Q(t)$. Let $b(t)$ and $r(t)$ be the number of blocked customers, and that of those who renege, respectively. Since the quantities $s(t)$ and $n(t)-b(t)-r(t)$ coincide in the long-run, then $Q$ can be rewritten as

$$
Q=\lim _{t \rightarrow \infty} \frac{n(t)-b(t)-r(t)}{n(t)}
$$

In what follows, we derive a closed-form expression for $Q$. We denote the system state by a random variable taking non-negative integer values representing the total number of customers in system (including those in service). We should first distinguish two sequences of probabilities that may describe the limiting state of our system. The first sequence is denoted by $p(i)$ and represents the steady state probability that the system is in state $i, 0 \leq i \leq K$, seen by an outside random observer (at a random instant). The second is denoted by $q(i)$ and represents the probability that an arriving customer finds the system in state $i$, which is in general different from $p(i), 0 \leq i \leq K$. The existence of both sequences is assured due to the ergodicity property of our system.

Clearly, the quantity $Q$ represents the probability in the infinite horizon for a new arrival customer to enter service, which involves as a consequence, the sequence $q(i)$. The latter is too difficult to be computed, however the sequence $p(i)$ is easy to derive as we shall detail below. Fortunately, we state for our system that the quantities $p(i)$ and $q(i)$ coincide for any state $i$. This is due to the well known PASTA property (Poisson Arrivals See Time Averages) also referred to as ROP (Random Observer Property), which holds here given that the arrival process is Poisson. The PASTA property is based on the memoryless property of the Poisson process, which allows to generate a sequence of arrivals that take a random look at the system. We refer the reader to Kleinrock [16] for further explanation, and Wolff [33] for a rigorous proof.

Let us now come back to Equation (3.1) by dividing both the numerator and the denominator in the right hand side over $t$. Computing $Q$ may be reduced thereafter to computing separately the ratios $n(t) / t, b(t) / t$ and $r(t) / t$ as $t$ goes to $\infty$. Recall that the mean number of customers per 
unit of time is $\lambda$. Hence in the long-run (as $t \rightarrow \infty$ ), the ratio $n(t) / t$ converges by construction to $\lambda$. As for the limit of $b(t) / t$ as $t \rightarrow \infty$, we may recognize it as the probability for a new arrival to be blocked times the mean arrival rate $\lambda$. So, it is the probability that a new arrival finds a full system times $\lambda$, namely the quantity $\lambda q(K)$, or equivalently $\lambda p(K)$. Let us now focus on the limit of $r(t) / t$ as $t$ goes to infinity. One may recognize this quantity as the mean number of reneging per unit of time seen by a random outside observer. Since it takes in average $1 / \gamma$ units of time for one customer waiting in queue to renege. Thus as $t \rightarrow \infty, r(t) / t$ converges to the mean number of customers in queue (in the distant future) times $\gamma$. Based on the previous analysis, $Q$ can be rewritten as follows.

$$
Q=1-p(K)-\frac{\gamma}{\lambda} \sum_{i=s+1}^{K}(i-s) p(i)
$$

To get explicitly the expression of $Q$, we move on to compute the stationary probabilities $p(i)$, for $0 \leq i \leq K$. In the usual way, we model our system as a finite continuous-time birthdeath process with discrete state space taking non-negative integer values ranging from 0 to $K$ and defined on a probability space. The birth rates are constant and equal to $\lambda$. The death rates are state-dependent; when moving from state $i$ to state $i-1$, the death rate is $i \mu$ for $1 \leq i \leq s$, and it is $s \mu+(i-s) \gamma$ for $s<i \leq K$. Under the stationary regime, we easily get a set of $K$ recursive equations relating $p(i)$ and $p(i+1)$ for $0 \leq i \leq K-1$. Proceeding to solve by iteration leads to

$$
p(i)=\frac{\lambda^{i}}{i ! \mu^{i}} p(0) \text { for } 0 \leq i \leq s, \text { and } p(i)=\frac{\lambda^{i}}{s ! \mu^{s} \prod_{j=1}^{i-s}(s \mu+j \gamma)} p(0) \text { for } s<i \leq K,
$$

where $p(0)$ is the steady state probability to have no customers in system, and obviously, $p(i)=0$ for $i>K$. Then, we couple the last set of equations with the probability conservation relation, i.e., $\sum_{i=0}^{\infty} p(i)=1$, to get

$$
p(0)=\left(\sum_{i=0}^{s} \frac{\lambda^{i}}{i ! \mu^{i}}+\frac{\lambda^{s}}{s ! \mu^{s}} \sum_{i=s+1}^{K} \frac{\lambda^{i-s}}{\prod_{j=1}^{i-s}(s \mu+j \gamma)}\right)^{-1},
$$

which determines all stationary probabilities. We still have to substitute them into Equations (3.2) to obtain $Q$. 


\section{Proof of First Order Monotonicity Property}

One may intuitively state that the performance measures $Q(t)$ and $Q$ increase with respect to the queue capacity $k$, keeping the parameters $\lambda, \mu, \gamma$ and $s$ constant. The idea is that, although adding more places in the waiting line may increase abandonments, it is clear that it could not deteriorate the performances we consider here. On the contrary, it allows for more customers to enter service. If not, it will at worst achieve an equal fraction of successful departures comparing to a system with less queue capacity. In this section, we rigorously prove these results using two different approaches. In Section 4.1, we prove using coupling arguments that $Q(t)$ and $Q$ increase in $k$ for a more general case, namely for a $G I / M / s / K+M$ queue. In Section 4.2, we consider our original system (the $M / M / s / K+M$ queue) and prove using an analytical approach that $Q$ increases in $k$.

\subsection{Sample Path Approach}

We start with a tangential development that will be of a great help to prove our main result. Let us relax some assumptions in our original system by considering a $G I / G I / s / K+M$ queue. We assume that interarrivals and service times are i.i.d., but we allow them to follow a general distribution. In Lemma (1), we present an interesting result about the relation between the performance measures of interest and the scheduling policy under which the system is working. For the rest of the paper, we denote by $\Pi$ the set of workconserving non-preemptive scheduling policies.

The following result in Lemma (1) can be seen as an extension of that in Theorem (2) of Jouini et al. [15]. In the latter, the author prove a conservation result for the probability of being lost in a queueing system with an infinite buffer size. Here, we prove the conservation result for the probability of being served by adding blocking (whenever the system is full).

Lemma 1 Consider a $G I / G I / s / K+M$ queue. Times before reneging are assumed to be i.i.d. and exponentially distributed. Then, the probability of being served $Q$ is constant for any workconserving non-preemptive scheduling policy.

Proof. The result is trivial for a queue with no capacity or with capacity 1 . In such cases, it is clear that the system behaves identically for any policy $\pi \in \Pi$, and as a consequence, $Q$ remains constant. Otherwise, for $k \geq 2$, we prove the result by coupling arguments. Consider two $G I / G I / s / K+M$ models, say Model 1 and Model 2. We assume that Model 1 and Model 2 
have identical parameters except for the scheduling policies. Model 1 and Model 2 are working under the policies $\pi_{1}$ and $\pi_{2}$, respectively, such that $\pi_{1} \in \Pi, \pi_{2} \in \Pi$, and $\pi_{1} \neq \pi_{2}$. Our approach is based on a single sample path. In both models, we create identical successive arrival epochs, as well as identical successive service times. However, since times before reneging are exponentially distributed, the decision for one customer to abandon the queue is not affected by his elapsed waiting time. This is stochastically equivalent to create randomly, for our sample path, a new maximum time of patience for each customer in queue after each selection for service epoch (or equivalently after each successful departure epoch). Assume that at time $t=0$ both systems are empty, and let work begins.

Both models behave identically until a busy period starts and the following situation occurs: a server becomes idle (after a service completion) and more than one customer are waiting in queue. Let $D_{i}$ be the epoch of that service completion (which occurs simultaneously in Model 1 and 2). For both models, let $n$ be the number of customers in queue just before $D_{i}, 2 \leq n \leq k$. At $D_{i}$, the idle server in Model 1 selects one customer from the queue who can be different from that selected by the same idle server in Model 2. However, the number of customers in queue goes down by 1 for both models, it becomes $n-1$. Recall that up to now, the number of blocked customers, as well as that of those who abandoned the queue, are identical for both models.

At the epoch $D_{i}$, we create for each customer, waiting in the queue of Model 1, a new patience time. Without altering distributions, since times before reneging are identically distributed, we create the same set of $n-1$ maximum patience times as in Model 1, and we assign them arbitrary to the customers waiting in Model 2. After $D_{i}$, three events are possible: one customer reneges, or a new arrival occurs, or a server becomes idle (service completion). Recall that by construction of our single sample path, these events occur simultaneously in both models. Assume now that the first event occurs, then the number of customers who abandon the queue goes up by 1 in both models and as a consequence remains identical for them. It is still also identical if another customer abandons the queue. In general, it is the case as long as there are customers waiting in queue. If not, both models will behave identically, anyway. Assume now that a new arrival occurs. Note that the number of customers in queue is the same in both models. If the queues are currently full, i.e., $k$ are waiting for service, then the new arrival will be blocked, and systems states remain unchanged. However, if at least there is 
one available space, hence, the number of customers in queue goes up by 1 in both models. Note that if another arrival occurs or that one customer abandons the queue, then, the number of customers in queue will increase by 1 (or remains unchanged if the system is full) or will decrease by 1 , respectively. The main conclusion is that the number of blocked customers, as well as that of those who abandon the queue will vary identically in both models.

Assume now that one server becomes idle. If the number of customers in queue is currently less or equal to 1 , it is obvious to see that policies $\pi_{1}$ and $\pi_{2}$ will select at the same time the unique available customer, if any. Otherwise, the busy period ends in both models, so both policies will again select identically new arrivals for service until the beginning of the next busy period. However, if the number of customers in queue is greater or equal to 2, the selected customer for service may be different from one model to another. As above, we create for the remaining set of waiting customers, the same set of patience time. Again, we can state that the number of blocked customers, as well as that of those who abandon the queue remains the same for both models.

Carrying on using the same arguments, we state that in a distant future, the number of blocked customers and that of those who renege in Model 1 coincide with those in Model 2. Since the number of arrivals are also equal for both models, the service level in terms of the fraction of successful departures is unchanged, $Q_{\pi 1}=Q_{\pi 2}$. This completes the proof.

Although the probability of being served is independent of the scheduling policy, the mean waiting time in queue for the served customers does depend on the scheduling policy. Jouini et al. [15] have proved the latter result when considering the particular case of a $G I / G I / s+M$ queue. They have also characterized the policies under which upper and lower bounds of the mean waiting time are achieved.

We should note however that the result in Lemma (1) does not hold if times before reneging are not i.i.d. and exponentially distributed, or if service times at any point during an arbitrary busy period are order of service dependent, we need to assume that no service needs are created or destroyed within the system: no renege in the midst of service, no forced idleness of servers, and so on.

In Lemma (2), we show that $Q$ is still unchanged for any workconserving scheduling policy (with preemption or not) if we further assume that service times are i.i.d. and exponentially 
distributed.

Lemma 2 Consider a $G I / M / s / K+M$ queue. Times before reneging are assumed to be i.i.d. and exponentially distributed. Then, the probability of being served $Q$ is constant for any workconserving scheduling policy.

Proof. We again show the result using coupling arguments. Based on a single sample path, we compare the quantity $Q$ for two identical $G I / M / s / K+M$ models, say Model 1 and Model 2 , working under two different scheduling policies $\pi_{1}$ and $\pi_{2}$, respectively. We assume that $\pi_{1}$ and $\pi_{2}$ are workconserving, and do not restrict them to be non-preemptive. We use a similar approach to that for Lemma (1). The only difference is only when an interruption of service occurs in one of the models. Note that just before the epoch of that event, both models are identical: all servers are busy, same number $n$ of customers in queue, same remaining service times, and same set of remaining times before reneging for waiting customers in queue. Without loss of generality, assume that in Model 1, a new arrival interrupts the service of a customer currently in service. Since service times are assumed to be exponentially distributed, then the remaining time for a service completion is not affected by the elapsed time in service. This allows us to create randomly, for our sample path, a new set of remaining service times for the customers currently in service ( $s$ customers in both models), and also a new set of patience time for waiting customers in queue ( $n$ customers in both models). Continuing the sample path comparison in the long run will subsequently show that $Q$ coincides for both models. This completes the proof of the lemma.

In Theorem (1), we show the main result of first order monotonicity for a $G I / M / s / K+M$ queue. The analysis resorts in part to the previous preliminary results of this section.

Theorem 1 Consider a $G I / M / s / K+M$ queue. Times before reneging are assumed to be i.i.d. and exponentially distributed. Then, probability of being served $Q$ is strictly increasing in the buffer size $k$.

Proof. To prove the result of the theorem, it suffices to compare the achieved $Q$ for the two following systems. The first, say Model 1 , is a $G I / M / s / K+M$ queue with $k$ waiting spaces. From Lemma (1), it does not restrict generality to assume that Model 1 works under the FCFS 
discipline of service. The second model is identical to the first in all parameters except that it has $k+1$ waiting spaces. From Lemma (2), the latter is equivalent, in terms of the achieved $Q$, to a $G I / M / s / K+M$ queue, say Model 2 , with $k+1$ waiting spaces and working under any preemptive workconserving policy. In summary, it is left to establish that the stationary probability of being served in Model 2 , say $Q_{2}$, is strictly greater than that in Model 1 , say $Q_{1}$.

The proof follows the sample path approach. Before proceeding to the details, let us characterize a specific preemptive workconserving policy, say $\pi$, under which Model 2 is operated. We divide the queue in Model 2 (with capacity $k+1$ ) into two virtual queues. The first, say queue 1 , has capacity $k$. The second, say queue 2 , has the remaining capacity, i.e., 1 . Upon arrival, a customer is addressed by one of the available servers, if any. If not, the customer must join one of the queues. We will specify the queue joining policy later. Customers in queue 1 have priority over customers in queue 2 in the sense that servers are handling customers belonging to queue 1 first. The priority rule is preemptive, which simply means that a server currently serving a customer pulled from queue 2, while a new arrival customer joins queue 1 , will interrupt this service and turn to queue 1 customer. Within each queue, customers are served in order of their arrival, that is, under the FCFS discipline.

Let us now couple Model 1 and 2 and let work begins. Both models behaves identically until the situation where in Model 1 all servers are busy, $k$ customers are waiting in queue and a new arrival occurs. Let us stop our clock temporarily. We denote that customer by the "low customer". Clearly, the "low customer" is blocked in Model 1 because the system is currently full, however, he joins the waiting line in Model 2. We assign him to queue 2 (with lower priority). Recall that up to now the number of customers served is identical in both models. Let our clock resumes ticking: arrivals, blocking, abandonments, as well as service completions will occur at the same epochs in both models until the busy period in system 1 ends (which occurs with probability 1 due to the ergodicity condition). We distinguish two possible cases for the "low customer": either he has meanwhile abandoned, or he is still waiting in queue 2. In the first case, both systems states become again identical. In the second case, i.e., if the "low customer" is still waiting, then we assign him to the server currently idle in Model 2. As long as the current idle period in Model 1 does not finish, we let the "low customer" stay in service. If the "low customer" finishes his service before that a new arrival occurs (at the same epoch in both models), therefore Model 2 will have one more service completion comparing to 
Model 1, and all events in both models become again identical. If not, that is if the idle period in Model 1 ends and the "low customer" has not successfully leaved Model 2, then we interrupt his service and we put him back in queue 2. The idea here from choosing the policy $\pi$ is to ensure an identical behavior, in both models, with regard to all customers except for the "low customers". Such customers are blocked (lost) in Model 1, however, they join queue 2 in Model 2.

From the previous arguments, one may easily deduce that $Q_{1} \leq Q_{2}$. Let us now proceed to establish that $Q_{1}<Q_{2}$. It is clear that one "low customer" at most may be present in Model 2 at a given observation moment. Let us further define a particular cycle duration referred to as the "low cycle". The "low cycle" starts when a "low customer" enters Model 2, and terminates upon the arrival of the next "low customer". The latter allows the following "low cycle" to start, and so on. The duration of a "low cycle" is given by the time it takes so that the "low customer" who starts the cycle either reneges or successfully finishes his service plus the time it takes starting from that epoch until the next "low customer" arrival epoch. Since the systems we consider here are stable, hence, any busy period in Model 1 ends with probability 1, i.e., its duration is finite $(<\infty)$. In addition, knowing that times before reneging are finite, we state that the "low cycle" duration is also finite. Furthermore, since interarrival times, times before reneging, as well as service times are i.i.d. and further independent of each others, it then follows that "low cycles" durations are also independent and identically distributed. Next, assuming the stationary regime and observing that there is a non-zero probability that a "low customer" finishes successfully his service within its corresponding "low cycle", it yields from the Law of Large Numbers that there is a non-zero proportion of "low customers" that will finish successfully their service. So, we state that the number of customers being served in Model 2 is strictly greater than that in Model 1. Finally, it is implied that the stationary probability of being served $Q$ is strictly increasing in the buffer size $k$. This completes the proof.

In a parallel to the proof of Theorem (1), we also state that the probability of being served under the transient regime, $Q(t)$, is an increasing function of $k$. Note that it is not necessarily strictly increasing in $k$ as it is the case for the quantity $Q$. 


\subsection{Analytical Approach}

In this section, we again consider our original $M / M / s / K+M$ queue described in Section 3.1. As shown in Section 3.2, a closed-form expression of the quantity $Q$ may be derived. This allows us to again prove the result of Theorem (1) using an analytical approach. The analysis we address in this section is in particular useful for the proof of the convexity result in Section 5. Before giving the details of the proof of the monotonicity property, we begin with some preliminary results by means of Properties (1) and (2). For the rest of the paper, an empty sum is being interpreted as zero, and an empty product is being interpreted as one.

Our objective is to show that $Q$ is strictly increasing in $k$ for an $M / M / s / K+M$ queue. To do so, we consider two models. The first is an $M / M / s / K+M$ queue with parameters $\lambda$, $\gamma, \mu, s$, and $k$ waiting spaces, $k \geq 0$. The second model is identical to the first however it has a larger buffer with $k+1$ waiting spaces. Recall that for our analysis, we do not need to specify the scheduling policy except that it is workconserving. Next, it suffices to show that the stationary probability of being served in the first model, say $Q_{k}$, is strictly lower than that in the second model, say $Q_{k+1}$. Or equivalently, if we introduce the sequence $\left\{U_{k}, k \geq 0\right\}$ defined as $U_{k}=Q_{k+1}-Q_{k}$, it remains for us to establish that $U_{k}>0$ for all $k \geq 0$. From Equation (3.2), $U_{k}$ can be rewritten as

$$
U_{k}=p_{k}(k+s)-p_{k+1}(k+s+1)+\frac{\gamma}{\lambda}\left(\sum_{i=s+1}^{k+s}(i-s) p_{k}(i)-\sum_{i=s+1}^{k+s+1}(i-s) p_{k+1}(i)\right) .
$$

The stationary probabilities are given by Equations (3.3) and (3.4). The subscripts are to indicate to which system the stationary probabilities are corresponding, either for the one with queue capacity $k$, or for that with queue capacity $k+1$. In Property (1), we state a useful relation between $U_{k}$ and $U_{k+1}$ for any non-negative integer $k$.

Property 1 For all $k \geq 0$, the following holds

$$
U_{k+1}=\frac{s ! \mu^{s} \sum_{i=0}^{s} \phi_{i}+\sum_{i=s+1}^{k+s} \rho_{i}}{s ! \mu^{s} \sum_{i=0}^{s} \phi_{i}+\sum_{i=s+1}^{k+s+2} \rho_{i}} \cdot \frac{\lambda}{s \mu+(k+2) \gamma} \cdot U_{k}
$$

where

$$
\phi_{i}=\frac{\lambda^{i}}{i ! \mu^{i}}, \text { for } i \geq 0, \text { and }, \rho_{i}=\frac{\lambda^{i}}{\prod_{j=1}^{i-s}(s \mu+j \gamma)}, \text { for } i \geq s+1
$$

Proof. The proof is provided in Appendix A. 
Note that proving Property (1) represents the "hard" part of the proof of the monotonicity result as well as that of the convexity result. One may verify that Equation (4.2) holds for different special cases. For instance, let us consider an infinite-server queue $M / M / s / K+M$ $(s \rightarrow \infty)$. Taking the limit in Relation (4.2) as $s$ goes to $\infty$ implies that $U_{k}=0$ for all nonnegative integer $k \geq 1$ (in addition from Equation (A.4) for example, we have $U_{0}=0$ ), which obviously agrees with the classical queueing results. The result also holds for an $M / M / s / K+M$ queue with infinitely impatient customers $(\gamma=\infty)$. In that case, the $M / M / s / K+M$ queue is equivalent to a loss system (without waiting space). Thus, it is easy to see that the quantity $Q_{k}$ does not depend on the buffer size $k$. So, $U_{k}=0$ for any $k \geq 0$, which agrees with Equation $(4.2)$.

Although we present in Property (2) an inequality that directly seems to be of independent interest, it is useful for forthcoming proofs of our results.

Property 2 Let $\lambda$ and $\mu$ be strictly positive reals and let $\left\{N_{s}, s \geq 1\right\}$ be a sequence defined as

$$
N_{s}=s \mu \sum_{i=0}^{s} \frac{\lambda^{i}}{i ! \mu^{i}}-\lambda \sum_{i=0}^{s-1} \frac{\lambda^{i}}{i ! \mu^{i}} .
$$

Then, $N_{s}>0$ for all $s \geq 1$.

Proof. The inequality holds by induction. We have $N_{1}=\mu>0$, then Property (2) holds for $s=1$. Assume now that $N_{s}>0$ for a given $s \geq 1$, and let us show that $N_{s+1}>0$. From Equation (4.4), $N_{s+1}$ can be written as

$$
\begin{aligned}
N_{s+1} & =(s+1) \mu \sum_{i=0}^{s+1} \frac{\lambda^{i}}{i ! \mu^{i}}-\lambda \sum_{i=0}^{s} \frac{\lambda^{i}}{i ! \mu^{i}} \\
& =s \mu \sum_{i=0}^{s} \frac{\lambda^{i}}{i ! \mu^{i}}+s \mu \frac{\lambda^{s+1}}{(s+1) ! \mu^{s+1}}+\mu \sum_{i=0}^{s+1} \frac{\lambda^{i}}{i ! \mu^{i}}-\lambda \sum_{i=0}^{s-1} \frac{\lambda^{i}}{i ! \mu^{i}}-\frac{\lambda^{s+1}}{s ! \mu^{s}} \\
& =N_{s}+\frac{s \lambda^{s+1}}{(s+1) ! \mu^{s}}-\frac{\lambda^{s+1}}{s ! \mu^{s}}+\mu \sum_{i=0}^{s+1} \frac{\lambda^{i}}{i ! \mu^{i}} \\
& =N_{s}-\frac{\lambda^{s+1}}{(s+1) ! \mu^{s}}+\mu \sum_{i=0}^{s} \frac{\lambda^{i}}{i ! \mu^{i}}+\frac{\lambda^{s+1}}{(s+1) ! \mu^{s}} \\
& =N_{s}+\mu \sum_{i=0}^{s} \frac{\lambda^{i}}{i ! \mu^{i}} .
\end{aligned}
$$

Using the induction assumption, it thus follows that $N_{s+1}>0$. Finally, we conclude that $N_{s}>0$ 
for all $s \geq 1$. This completes the proof.

In Theorem 2, we state the main result of this section. Having Properties (1) and (2), we are now ready to establish the first order monotonicity property of the probability of being served, $Q$, with respect to the buffer size $k$.

Theorem 2 Consider an $M / M / s / K+M$ queue. Times before reneging are assumed to be i.i.d. and exponentially distributed. Then, $Q$ is strictly increasing in the buffer size $k$.

Proof. As explained in the beginning of this section, proving the theorem is equivalent to proving that $U_{k}$ is strictly positive for $k \geq 0$. Keeping the parameters $\lambda, \mu, s$ and $\gamma$ constant, the result holds by induction on $k$.

Let us establish our claim for the first rank, $k=0$. The quantity $U_{0}$ is given by $U_{0}=Q_{1}-Q_{0}$, where $Q_{0}$ and $Q_{1}$ are the probabilities of being served for the $M / M / s / s+M$ (no waiting space) and $M / M / s / s+1+M$ (single waiting space) systems, respectively. Using Equation (3.2), the probability $Q_{0}$ is given by $Q_{0}=1-p_{0}(s)$, where $p_{0}(s)$ is the stationary probability to have $s$ customers in the $M / M / s / s+M$ system. As for $Q_{1}$, it is given by $Q_{1}=1-\frac{\lambda+\gamma}{\lambda} p_{1}(s+1)$, where $p_{1}(s+1)$ is the stationary probability to have $s+1$ customers in the $M / M / s / s+1+M$ system.

From Equations (3.3) and (3.4), we get

$$
Q_{0}=1-\frac{\frac{\lambda^{s}}{s ! \mu^{s}}}{\sum_{i=0}^{s} \frac{\lambda^{i}}{i ! \mu^{i}}}
$$

and,

$$
Q_{1}=1-\frac{\lambda+\gamma}{s \mu+\gamma} \frac{\frac{\lambda^{s}}{s ! \mu^{s}}}{\sum_{i=0}^{s} \frac{\lambda^{i}}{i ! \mu^{i}}+\frac{\lambda^{s}}{s ! \mu^{s}} \frac{\lambda}{s \mu+\gamma}}
$$

Therefore,

$$
U_{0}=\frac{\lambda^{s}}{s ! \mu^{s} \sum_{i=0}^{s} \frac{\lambda^{i}}{i ! \mu^{i}}}-\frac{\lambda^{s+1}+\lambda^{s} \gamma}{s ! \mu^{s}(s \mu+\gamma) \sum_{i=0}^{s} \frac{\lambda^{i}}{i ! \mu^{i}}+\lambda^{s+1}} .
$$

To prove that $U_{0}>0$, we consider $U_{0}$ as a real function of $\gamma$, for $\gamma \geq 0$, and we study the sign of $U_{0}(\gamma)$. It is clear that $U_{0}$ has the property to be continuous and derivable in $\gamma$. Taking the 
derivative, $U_{0}^{\prime}$, of $U_{0}$ in $\gamma$ leads to

$$
\begin{aligned}
& U_{0}^{\prime}(\gamma)=-\frac{s ! \mu^{s} s \mu \sum_{i=0}^{s} \frac{\lambda^{i}}{i ! \mu^{i}}+s ! \mu^{s} \gamma \sum_{i=0}^{s} \frac{\lambda^{i}}{i ! \mu^{i}}+\lambda^{s+1}-(\lambda+\gamma) s ! \mu^{s} \sum_{i=0}^{s} \frac{\lambda^{i}}{i ! \mu^{i}}}{\left(s ! \mu^{s}(s \mu+\gamma) \sum_{i=0}^{s} \frac{\lambda^{i}}{i ! \mu^{i}}+\lambda^{s+1}\right)^{2}} \cdot \lambda^{s} \\
& =-\frac{s ! \mu^{s} s \mu \sum_{i=0}^{s} \frac{\lambda^{i}}{i ! \mu^{i}}+s ! \mu^{s} \gamma \sum_{i=0}^{s} \frac{\lambda^{i}}{i ! \mu^{i}}+\lambda^{s+1}-(\lambda+\gamma)\left(s ! \mu^{s} \sum_{i=0}^{s-1} \frac{\lambda^{i}}{i ! \mu^{i}}+\lambda^{s}\right)}{\left(s ! \mu^{s}(s \mu+\gamma) \sum_{i=0}^{s} \frac{\lambda^{i}}{i ! \mu^{i}}+\lambda^{s+1}\right)^{2}} \cdot \lambda^{s} \\
& =-\frac{s ! \mu^{s} s \mu \sum_{i=0}^{s} \frac{\lambda^{i}}{i ! \mu^{i}}+s ! \mu^{s} \gamma \sum_{i=0}^{s-1} \frac{\lambda^{i}}{i ! \mu^{i}}+\lambda^{s} \gamma+\lambda^{s+1}}{\left(s ! \mu^{s}(s \mu+\gamma) \sum_{i=0}^{s} \frac{\lambda^{i}}{i ! \mu^{i}}+\lambda^{s+1}\right)^{2}} \cdot \lambda^{s} \\
& +\frac{\lambda^{s+1}+\lambda s ! \mu^{s} \sum_{i=0}^{s-1} \frac{\lambda^{i}}{i ! \mu^{i}}+\lambda^{s} \gamma+s ! \mu^{s} \gamma \sum_{i=0}^{s-1} \frac{\lambda^{i}}{i ! \mu^{i}}}{\left(s ! \mu^{s}(s \mu+\gamma) \sum_{i=0}^{s} \frac{\lambda^{i}}{i ! \mu^{i}}+\lambda^{s+1}\right)^{2}} \cdot \lambda^{s} \\
& =-\lambda^{s} s ! \mu^{s} \frac{s \mu \sum_{i=0}^{s} \frac{\lambda^{i}}{i ! \mu^{i}}-\lambda \sum_{i=0}^{s-1} \frac{\lambda^{i}}{i ! \mu^{i}}}{\left(s ! \mu^{s}(s \mu+\gamma) \sum_{i=0}^{s} \frac{\lambda^{i}}{i ! \mu^{i}}+\lambda^{s+1}\right)^{2}} \text {. }
\end{aligned}
$$

Using the notation in Equation (4.4), $U_{0}^{\prime}(\gamma)$ can be rewritten as

$$
U_{0}^{\prime}(\gamma)=\frac{-\lambda^{s} s ! \mu^{s}}{\left(s ! \mu^{s}(s \mu+\gamma) \sum_{i=0}^{s} \frac{\lambda^{i}}{i ! \mu^{i}}+\lambda^{s+1}\right)^{2}} \cdot N_{s}
$$

Applying now Property (2) for $s$ strictly positive integer, and, for $\lambda$ and $\mu$ strictly positive reals, we easily see that $U_{0}^{\prime}(\gamma)<0$. Then, $U_{0}$ is a strictly decreasing function in $\gamma$, for $\gamma \geq 0$. Hence, it follows that

$$
U_{0}(\gamma)>\lim _{\gamma \rightarrow+\infty} U_{0}(\gamma), \text { for } \gamma \geq 0
$$

Observing that

$$
\lim _{\gamma \rightarrow+\infty} U_{0}(\gamma)=\frac{\lambda^{s}}{s ! \mu^{s} \sum_{i=0}^{s} \frac{\lambda^{i}}{i ! \mu^{i}}}-\frac{\lambda^{s+1}+\lambda^{s} \gamma}{s ! \mu^{s}(s \mu+\gamma) \sum_{i=0}^{s} \frac{\lambda^{i}}{i ! \mu^{i}}+\lambda^{s+1}}=0
$$

we deduce that $U_{0}(\gamma)>0$ for $\gamma \geq 0$. Thereafter, our claim is true for the first rank $k=0$.

Let us consider $k \geq 0$ and assume that our claim is true for the rank $k$, i.e., $U_{k}>0$. Let us now prove that our claim is true for the rank $k+1$. This is a direct consequence of Property (1). For $s \geq 1, \lambda, \mu>0$ and $\gamma \geq 0$, we state using Property (1) that $U_{k+1}$ is the product of $U_{k}$ and a strictly positive real. So, $U_{k+1}>0$. Finally, we conclude that $U_{k}>0$ for $k \geq 0$, which completes the proof of the theorem. 


\section{Proof of Second Order Monotonicity Property}

In this section, we investigate the second order property of monotonicity (of the probability of being served) in the queue capacity. First, we prove using a simple counterexample that the transient probability of being served, $Q(t)$, is not concave in $k$. Second, we state our main result in Theorem (3) about the concavity property. Finally, we present some numerical illustrations of that result.

To prove the non-concavity of $Q(t)$ as a function of $k$, we consider three $M / M / 1 / K+M$ queues denoted by Model 1, Model 2 and Model 3. Assume the discipline of service to be FCFS. The models are identical in all parameters except for the buffer size. Specifically, Models 1, 2 and 3 contain 1, 2 and 3 waiting spaces, respectively. During an interval of time $[0, t]$, we denote the transient probability of being served for Model 1 by $Q_{1}(t)$. We denote those for Model 2 and 3 by $Q_{2}(t)$ and $Q_{3}(t)$, respectively. In what follows, we construct one possible sample path which shows that the transient probability of being served is not concave in $k$. In mathematical terms, it consists to find an instant $t$ such that $Q_{3}(t)-Q_{2}(t)>Q_{2}(t)-Q_{1}(t)$.

Initially, the models are empty. Now, let work begins. All models behave identically until the situation where in each model the unique available server is busy and there is one waiting customer in queue, say $A_{1}$. Thereafter, assume that one arrival, say $A_{2}$, occurs before a service completion or an abandonment. Note that this event occurs with a non-zero probability. The customer $A_{2}$ is blocked in Model 1, whereas he joins the queue in Models 2 and 3. Assume also that the next event is an arrival denoted by $A_{3}$. The customer $A_{3}$ is blocked in Models 1 and 2, however he joins the queue in Model 3. Next, assume that $A_{2}$ abandons the queue, which occurs simultaneously in Models 2 and 3. Then, assume that $A_{1}$ in all models and $A_{3}$ in Model 3 finish their service and successfully leave the systems. Let $t_{A_{3}}$ be the epoch of the departure of $A_{3}$. So, we state that during $\left[0, t_{A_{3}}\right]$, the number of served customers in Model 1 is equal to that in Model 2. However, there is one served customer in more in Model 3 compared to the other models. In other words, $Q_{3}(t)>Q_{2}(t)$ and $Q_{1}(t)=Q_{2}(t)$, which leads to the inequality $Q_{3}(t)-Q_{2}(t)>Q_{2}(t)-Q_{1}(t)$ and closes the discussion.

Turning now to the concavity of the stationary quantity $Q$ as a function of $k$, we present the following theorem.

Theorem 3 Consider an $M / M / s / K+M$ queue. Times before reneging are assumed to be i.i.d. and exponentially distributed. Then, $Q$ is a strictly concave function in the buffer size $k$. 
Proof. Let us again consider three $M / M / s / K+M$ queues denoted by Model 1, 2 and 3. All models are identical in all parameters except in the buffer size. In Model 1, there are $k$ waiting spaces. However, Model 2 and Model 3 have $k+1$ and $k+2$ waiting spaces, respectively. We do not need here to specify the scheduling policy except that it is workconserving. For Model 1,2 and 3 , we denote by $Q_{k}, Q_{k+1}$ and $Q_{k+2}$ the stationary probabilities of being served, respectively. Following this introduction, one may easily see that proving our theorem is equivalent to proving that $U_{k}=Q_{k+1}-Q_{k}$ is strictly greater than $U_{k+1}=Q_{k+2}-Q_{k+1}$, for all $k \geq 0$. In other terms, it remains to prove that the sequence $\left\{U_{k}, k \geq 0\right\}$ is strictly decreasing. Knowing from Theorem (2) that $U_{k}>0$, for $k \geq 0$, it suffices thereafter to show that $\frac{U_{k+1}}{U_{k}}<1$, for $k \geq 0$. From Equation (4.2), we have

$$
\frac{U_{k+1}}{U_{k}}=\frac{s ! \mu^{s} \sum_{i=0}^{s} \phi_{i}+\sum_{i=s+1}^{k+s} \rho_{i}}{s ! \mu^{s} \sum_{i=0}^{s} \phi_{i}+\sum_{i=s+1}^{k+s+2} \rho_{i}} \cdot \frac{\lambda}{s \mu+(k+2) \gamma}, \text { for } k \geq 0,
$$

which may be rewritten, for $k \geq 0$, as

$$
\frac{U_{k+1}}{U_{k}}=\frac{\left(\left(s ! \mu^{s} \sum_{i=0}^{s-1} \phi_{i}\right) \times \frac{\lambda}{s \mu+(k+2) \gamma}\right)+\left(\left(\lambda^{s}+\sum_{i=s+1}^{k+s} \rho_{i}\right) \times \frac{\lambda}{s \mu+(k+2) \gamma}\right)}{s ! \mu^{s} \sum_{i=0}^{s} \phi_{i}+\sum_{i=s+1}^{k+s+2} \rho_{i}} .
$$

From the one hand, Property (2) leads to

$$
\lambda \sum_{i=0}^{s-1} \phi_{i}<(s \mu+(k+1) \gamma) \sum_{i=0}^{s} \phi_{i}
$$

Hence,

$$
\left(s ! \mu^{s} \sum_{i=0}^{s-1} \phi_{i}\right) \times \frac{\lambda}{s \mu+(k+1) \gamma}<s ! \mu^{s} \sum_{i=0}^{s} \phi_{i} .
$$

From the other hand, we have for all $i$, such that $i<k+s+1$,

$$
\frac{\lambda}{s \mu+(k+2) \gamma}<\frac{\lambda}{s \mu+(i-s+1) \gamma},
$$

which implies

$$
\frac{\lambda}{s \mu+(k+2) \gamma} \rho_{i}<\frac{\lambda}{s \mu+(i-s+1) \gamma} \rho_{i}=\rho_{i+1} .
$$


Summing Equation (5.6) on all $i, s+1 \leq i \leq k+s$, we get

$$
\frac{\lambda}{s \mu+(k+2) \gamma} \sum_{i=s+1}^{k+s} \rho_{i}<\sum_{i=s+1}^{k+s} \rho_{i+1}=\sum_{i=s+2}^{k+s+1} \rho_{i} .
$$

Next, observing that

$$
\frac{\lambda^{s+1}}{s \mu+(k+2) \gamma}<\frac{\lambda^{s+1}}{s \mu+\gamma}=\rho_{s+1}, \text { for } k \geq 0
$$

the summation of both Inequalities (5.7) and (5.8) leads to

$$
\left(\lambda^{s}+\sum_{i=s+1}^{k+s} \rho_{i}\right) \times \frac{\lambda}{s \mu+(k+2) \gamma}<\sum_{i=s+1}^{k+s+1} \rho_{i}<\sum_{i=s+1}^{k+s+2} \rho_{i} .
$$

Finally, it remains to apply Relations (5.4) and (5.9) back into Relation (5.2), to state that $\frac{U_{k+1}}{U_{k}}<1$. This completes the proof of the theorem.

To get some numerical illustrations of our results, we consider various $M / M / s / K+M$ models by taking a broad range of parameters values. The service rate is unchanged for all chosen examples, $\mu=1$. The values of the reneging rate are $0.5,1$ and 2 . The number of servers are $1,2,3,5,10,15,50,70$ and 100 . To vary the "servers utilization" calculated as $\lambda / s \mu$, we consider $\lambda=1.8$ for $s=1,2$ and $3 ; \lambda=8$ for $s=5,10$ and $15 ; \lambda=60$ for $s=50,70$ and 100 . For each set of the previous values, the buffer size is ranging from 0 to 30 . The detailed results are presented in Tables 1, 2 and 3 of Appendix B.

From the numerical results, we underline the following comments. As expected from Theorems (2) and (3), $Q$ is increasing and concave in $k$ keeping all remaining parameters constant. One may see that there is no need to go beyond a buffer size around 10 to approximately reach the maximum of the probability of being served (reached within an infinite buffer size). Starting from a system with no waiting space, most of the improvements are achieved by adding two places in the buffer. Obviously, we also see that $Q$ is decreasing with respect to the abandonment rate $\gamma$. The reason is simply that the probability to abandon the queue is increasing in the abandonment rate. Furthermore, for a fixed server utilization, large systems allow to achieve higher service levels. This does not seem at odds with known results, it is due to the pooling effect. 


\section{Conclusions}

In this paper, we considered a queueing system with reneging and finite buffer size. The model is of interest for the modeling in practice of several systems with impatient customers, such as call centers. We investigated monotonicity results of the probability of being served with respect to the buffer size. These results are helpful when addressing optimizations issues. We considered both transient and stationary quantities of the performance of interest. Under the transient regime, we proved that it is an increasing and non-concave function of the buffer size. Under the stationary regime, we proved that it is strictly increasing and concave in the buffer size.

As a topic for future research, it would be interesting to investigate in a similar fashion as here, the convexity properties of the performance measure as a function of other parameters such as the arrival rate, service rate, reneging rate, and number of servers.

\section{Acknowledgements}

The authors wish to thank Ger Koole and Mohamed Salah Aguir for their helpful discussions to improve this paper.

\section{References}

1. C. J. Ancker and A. Gafarian. Queueing with Impatient Customers Who Leave at Random. Journal of Industrial Engineering, 13:84-90, 1962.

2. M. Armony, E. Plambeck, and S. Seshadri. Convexity Properties and Comparative Statics for an M/M/S Queue with Impatient Customers: Why You Shouldn't Shout at the DMV. 2005. Working paper. New York University.

3. F. Baccelli and G. Hebuterne. On Queues With Impatient Customers. Performance'81 North-Holland Publishing Company, pages 159-179, 1981.

4. A.W. Berger and W. Whitt. Comparisons of Multi-Server Queues with Finite Waiting Rooms. Stochastic Models, 8:719-732, 1992.

5. P.P. Bhattacharya and A. Ephremides. Stochastic Monotonicity Properties of Multiserver Queues with Impatient Customers. Journal of Applied Probability, 28:673-682, 1991. 
6. A. Brandt and M. Brandt. Assymptotic Results and a Markovian Approximation for the M(n)/M(n)/C + GI System. Queueing Systems, 41:73-94, 2002.

7. O. Garnett, A. Mandelbaum, and M. Reiman. Designing a Call Center with Impatient Customers. Manufacturing $\mathcal{E}$ Service Operations Management, 4:208-227, 2002.

8. W. Grassmann. The Convexity of the Mean Queue Size of the M/M/C Queue with Respect to the Traffic Intensity. Journal of Applied Probability, 20:916-919, 1987.

9. A. Harel. Convexity of the Erlang Loss Formula. Operations Research, 38:499-505, 1990.

10. A. Harel. Convexity Results for Single-Server Queue and for Multiserver Queues with Constant Service Times. Journal of Applied Probability, 27:465-468, 1990.

11. A. Harel and P. Zipkin. The Convexity of a General Performance Measure for Multiserver Queues. Journal of Applied Probability, 24:725-736, 1987.

12. A. Harel and P. H. Zipkin. Strong Convexity Results for Queueing Systems. Operations Research, 35:405-418, 1987.

13. D. L. Jagerman. Some Properties of the Erlang Loss Function. The Bell System Technical Journal, 53:525-551, 1974.

14. A. A. Jagers and E. A. van Doorn. Convexity of Functions Which Are Generalizations of the Erlang Loss Function and the Erlang Delay Function. SIAM Review, 33:281-282, 1991.

15. O. Jouini, A. Pot, G. Koole, and Y. Dallery. Online Scheduling Policies for Multiclass Call Centers with Impatient Customers. European Journal of Operational Research, 207:258268,2010 .

16. L. Kleinrock. Queueing Systems, Theory, volume I. A Wiley-Interscience Publication, 1975.

17. G. Koole. Convexity in Tandem Queues. Probability in the Engineering and Informational Sciences, 18:13-31, 2004.

18. G. Koole, M. Nuyens, and R. Righter. The Effect of Service Time Variability on Maximum Queue Lengths in MX/G/1 Queues. Journal of Applied Probability, 42, 2005. To appear. 
19. G. Koole and A. Pot. A Note on Profit Maximization and Monotonicity for Inbound Call Centers. 2005. Working paper. Vrije Universiteit Amsterdam.

20. H. L. Lee and M. A. Cohen. A Note on the Convexity of Performance Measures of M/M/C Queueing Systems. Journal of Applied Probability, 20:920-923, 1983.

21. L. Meister and J. G. Shanthikumar. Concavity of the Throughput of Tandem Queueing System with Finite Buffer Storage Space. Advances In Applied Probability, 22:764-767, 1990.

22. E. J. Messerli. Proof of a Convexity Property of the Erlang B Formula. The Bell System Technical Journal, 51:951-553, 1972.

23. R. Nagarajan and D. Towsley. A Note on the Convexity of the Probability of a Full Buffer in the M/M/1/K queue. 1992. CMPSCI Technical Report TR 92-85, MIT.

24. A. Pacheco. Second-Order Properties of the Loss Probability in M/M/s/s + c Systems. Queueing Systems, 15:309-324, 1994.

25. M. P. Pierson and W. Whitt. A Statistically-Fit Markovian Approximation of a Basic Call-Center Model. 2006. Working paper, Columbia University.

26. M. Shaked and J. G. Shanthikumar. Stochastic Convexity and Its Applications. Advances in Applied Probability, 20:427-446, 1988.

27. J. G. Shanthikumar. Stochastic Majorization of Random Variables with Proportional Equilibrium Rate. Advances in Applied Probability, 19:854-872, 1987.

28. H. Y. Tu and H. Kumin. A Convexity Result for a Class of GI/G/1 Queueing Systems. Operations Research, 31:948-950, 1983.

29. A.R. Ward and P.W. Glynn. A Diffusion Approximation for a Markovian Queue with Reneging. Queueing Systems, 43:103-128, 2003.

30. R. R. Weber. On the Marginal Benefit of Adding Servers to G/GI/m Queues. Management Science, 26:946-951, 1980.

31. R. R. Weber. A Note on Waiting Times in Single Server Queues. Operations Research, 31:950-951, 1983. 
32. W. Whitt. Sensitivity of Performance in the Erlang-A Queueing Model to Changes in the Model Parameters. Operations Research, 54:247-260, 2006.

33. R.W. Wolff. Poisson Arrivals See Time Averages. Operations Research, 30:223-231, 1982.

34. D. D. Yao and J. G. Shanthikumar. The Optimal Input Rate to a System of Manufacturing Cells. Information Systems and Operational Research, 25:57-65, 1987.

35. S. Zeltyn and A. Mandelbaum. Call Centers with Impatient Customers: Many-Servers Asymptotics of the M/M/n+G Queue. Queueing Systems, 51:361-402, 2005.

\section{Appendixes}

\section{A Proof of Property (1)}

In this appendix, we prove the result in Property (1). Using the notations in Equation (4.3), the stationary probabilities for an $M / M / s / s+k+M$ queue ( $k$ extra waiting lines) given in Equations (3.3) and (3.4) may be rewritten as

$$
\begin{gathered}
p_{k}(i)=\phi_{i} \times p(0), \quad \text { for } 0 \leq i \leq s, \\
p_{k}(i)=\rho_{i} \times p(0), \quad \text { for } s<i \leq s+k,
\end{gathered}
$$

and

$$
p_{k}(0)=\frac{s ! \mu^{s}}{s ! \mu^{s} \sum_{i=0}^{s} \phi_{i}+\sum_{i=s+1}^{s+k} \rho_{i}} .
$$

Substituting them into Equation (4.1) yields, for $k \geq 0$, to

$$
\begin{aligned}
U_{k}=\left(\frac{\rho_{k+s}}{s ! \mu^{s} \sum_{i=0}^{s} \phi_{i}+\sum_{i=s+1}^{k+s} \rho_{i}}-\frac{\rho_{k+s+1}}{s ! \mu^{s} \sum_{i=0}^{s} \phi_{i}+\sum_{i=s+1}^{k+s+1} \rho_{i}}\right) \\
+\frac{\gamma}{\lambda}\left(\frac{\sum_{i=s+1}^{k+s}(i-s) \rho_{i}}{s ! \mu^{s} \sum_{i=0}^{s} \phi_{i}+\sum_{i=s+1}^{k+s} \rho_{i}}-\frac{\sum_{i=s+1}^{k+s+1}(i-s) \rho_{i}}{s ! \mu^{s} \sum_{i=0}^{s} \phi_{i}+\sum_{i=s+1}^{k+s+1} \rho_{i}}\right) .
\end{aligned}
$$


Or equivalently with some algebra

$$
\begin{aligned}
U_{k}\left(s ! \mu^{s}\right. & \left.\sum_{i=0}^{s} \phi_{i}+\sum_{i=s+1}^{k+s+1} \rho_{i}\right)=\rho_{k+s}\left(1+\frac{\rho_{k+s+1}}{s ! \mu^{s} \sum_{i=0}^{s} \phi_{i}+\sum_{i=s+1}^{k+s} \rho_{i}}\right)-\rho_{k+s+1} \\
& +\frac{\gamma}{\lambda}\left(\sum_{i=s+1}^{k+s}(i-s) \rho_{i}\left(1+\frac{\rho_{k+s+1}}{s ! \mu^{s} \sum_{i=0}^{s} \phi_{i}+\sum_{i=s+1}^{k+s} \rho_{i}}\right)-\sum_{i=s+1}^{k+s+1}(i-s) \rho_{i}\right) \\
= & \rho_{k+s}+\frac{\rho_{k+s} \rho_{k+s+1}}{s ! \mu^{s} \sum_{i=0}^{s} \phi_{i}+\sum_{i=s+1}^{k+s} \rho_{i}}-\rho_{k+s+1} \\
& +\frac{\gamma}{\lambda}\left(-(k+1) \rho_{k+s+1}+\frac{\rho_{k+s+1}}{s ! \mu^{s} \sum_{i=0}^{s} \phi_{i}+\sum_{i=s+1}^{k+s} \rho_{i}} \sum_{i=s+1}^{k+s}(i-s) \rho_{i}\right) \\
= & \rho_{k+s}+\rho_{k+s+1}\left(-1+\frac{\rho_{k+s}}{s ! \mu^{s} \sum_{i=0}^{s} \phi_{i}+\sum_{i=s+1}^{k+s} \rho_{i}}-\frac{(k+1) \gamma}{\lambda}+\frac{\gamma}{\lambda} \frac{\sum_{i=s+1}^{k+s}(i-s) \rho_{i}}{s ! \mu^{s} \sum_{i=0}^{s} \phi_{i}+\sum_{i=s+1}^{k+s} \rho_{i}}\right) .
\end{aligned}
$$

Calculating further gives

$$
\begin{aligned}
& U_{k}\left(s ! \mu^{s} \sum_{i=0}^{s} \phi_{i}+\sum_{i=s+1}^{k+s+1} \rho_{i}\right)\left(s ! \mu^{s} \sum_{i=0}^{s} \phi_{i}+\sum_{i=s+1}^{k+s} \rho_{i}\right)=\rho_{k+s}\left(s ! \mu^{s} \sum_{i=0}^{s} \phi_{i}+\sum_{i=s+1}^{k+s} \rho_{i}\right) \\
& +\rho_{k+s+1}\left(\rho_{k+s}-\left(s ! \mu^{s} \sum_{i=0}^{s} \phi_{i}+\sum_{i=s+1}^{k+s} \rho_{i}\right)-\frac{(k+1) \gamma}{\lambda}\left(s ! \mu^{s} \sum_{i=0}^{s} \phi_{i}+\sum_{i=s+1}^{k+s} \rho_{i}\right)+\frac{\gamma}{\lambda} \sum_{i=s+1}^{k+s}(i-s) \rho_{i}\right) \\
& =\rho_{k+s}\left(s ! \mu^{s} \sum_{i=0}^{s} \phi_{i}+\sum_{i=s+1}^{k+s} \rho_{i}\right)+\rho_{k+s+1}\left(\rho_{k+s}-\left(1+\frac{(k+1) \gamma}{\lambda}\right)\left(s ! \mu^{s} \sum_{i=0}^{s} \phi_{i}+\sum_{i=s+1}^{k+s} \rho_{i}\right)+\frac{\gamma}{\lambda} \sum_{i=s+1}^{k+s}(i-s) \rho_{i}\right) .
\end{aligned}
$$

Observing that $\rho_{k+s+1}=\frac{\lambda}{s \mu+(k+1) \gamma} \rho_{k+s}$, for $k \geq 0$, we may write

$$
\begin{gathered}
U_{k}\left(s ! \mu^{s} \sum_{i=0}^{s} \phi_{i}+\sum_{i=s+1}^{k+s+1} \rho_{i}\right)\left(s ! \mu^{s} \sum_{i=0}^{s} \phi_{i}+\sum_{i=s+1}^{k+s} \rho_{i}\right) \frac{1}{\rho_{k+s}} \\
=\left(s ! \mu^{s} \sum_{i=0}^{s} \phi_{i}+\sum_{i=s+1}^{k+s} \rho_{i}+\rho_{k+s+1}\right)-\frac{\lambda+(k+1) \gamma}{s \mu+(k+1) \gamma}\left(s ! \mu^{s} \sum_{i=0}^{s} \phi_{i}+\sum_{i=s+1}^{k+s} \rho_{i}\right)+\frac{\gamma}{s \mu+(k+1) \gamma} \sum_{i=s+1}^{k+s}(i-s) \rho_{i} \\
=\left(s ! \mu^{s} \sum_{i=0}^{s} \phi_{i}+\sum_{i=s+1}^{k+s+1} \rho_{i}\right)-\left(s ! \mu^{s} \sum_{i=0}^{s} \phi_{i}+\sum_{i=s+1}^{k+s} \rho_{i}\right) \\
\quad-\frac{\lambda-s \mu}{s \mu+(k+1) \gamma}\left(s ! \mu^{s} \sum_{i=0}^{s} \phi_{i}+\sum_{i=s+1}^{k+s} \rho_{i}\right)+\frac{\gamma}{s \mu+(k+1) \gamma} \sum_{i=s+1}^{k+s}(i-s) \rho_{i} .
\end{gathered}
$$

Simplifying Equation (A.7) implies the following relation

$$
\begin{aligned}
& U_{k}\left(s ! \mu^{s} \sum_{i=0}^{s} \phi_{i}+\sum_{i=s+1}^{k+s+1} \rho_{i}\right)\left(s ! \mu^{s} \sum_{i=0}^{s} \phi_{i}+\sum_{i=s+1}^{k+s} \rho_{i}\right) \frac{1}{\rho_{k+s}} \\
& =\rho_{k+s+1}-\frac{\lambda-s \mu}{s \mu+(k+1) \gamma}\left(s ! \mu^{s} \sum_{i=0}^{s} \phi_{i}+\sum_{i=s+1}^{k+s} \rho_{i}\right)+\frac{\gamma}{s \mu+(k+1) \gamma} \sum_{i=s+1}^{k+s}(i-s) \rho_{i} .
\end{aligned}
$$


For the rank $k+1$, Relation (A.8) becomes

$$
\begin{gathered}
U_{k+1}\left(s ! \mu^{s} \sum_{i=0}^{s} \phi_{i}+\sum_{i=s+1}^{k+s+2} \rho_{i}\right)\left(s ! \mu^{s} \sum_{i=0}^{s} \phi_{i}+\sum_{i=s+1}^{k+s+1} \rho_{i}\right) \frac{1}{\rho_{k+s+1}} \\
=\rho_{k+s+2}-\frac{\lambda-s \mu}{s \mu+(k+2) \gamma}\left(s ! \mu^{s} \sum_{i=0}^{s} \phi_{i}+\sum_{i=s+1}^{k+s+1} \rho_{i}\right)+\frac{\gamma}{s \mu+(k+2) \gamma} \sum_{i=s+1}^{k+s+1}(i-s) \rho_{i} \\
=\frac{\lambda}{s \mu+(k+2) \gamma} \rho_{k+s+1}-\frac{\lambda-s \mu}{s \mu+(k+2) \gamma}\left(s ! \mu^{s} \sum_{i=0}^{s} \phi_{i}+\sum_{i=s+1}^{k+s} \rho_{i}+\rho_{k+s+1}\right) \\
\quad+\frac{\gamma}{s \mu+(k+2) \gamma} \sum_{i=s+1}^{k+s}(i-s) \rho_{i}+\frac{(k+1) \gamma}{s \mu+(k+2) \gamma} \rho_{k+s+1} .
\end{gathered}
$$

Hence,

$$
\begin{aligned}
& U_{k+1}\left(s ! \mu^{s} \sum_{i=0}^{s} \phi_{i}+\sum_{i=s+1}^{k+s+2} \rho_{i}\right)\left(s ! \mu^{s} \sum_{i=0}^{s} \phi_{i}+\sum_{i=s+1}^{k+s+1} \rho_{i}\right) \frac{1}{\rho_{k+s+1}} \\
& =\frac{s \mu+(k+1) \gamma}{s \mu+(k+2) \gamma} \rho_{k+s+1}-\frac{\lambda-s \mu}{s \mu+(k+2) \gamma}\left(s ! \mu^{s} \sum_{i=0}^{s} \phi_{i}+\sum_{i=s+1}^{k+s} \rho_{i}\right)+\frac{\gamma}{s \mu+(k+2) \gamma} \sum_{i=s+1}^{k+s}(i-s) \rho_{i} .
\end{aligned}
$$

Multiplying both sides in Equation (A.10) by $\frac{s \mu+(k+2) \gamma}{s \mu+(k+1) \gamma}$ implies

$$
\begin{aligned}
& U_{k+1}\left(s ! \mu^{s} \sum_{i=0}^{s} \phi_{i}+\sum_{i=s+1}^{k+s+2} \rho_{i}\right)\left(s ! \mu^{s} \sum_{i=0}^{s} \phi_{i}+\sum_{i=s+1}^{k+s+1} \rho_{i}\right) \frac{1}{\rho_{k+s+1}} \cdot \frac{s \mu+(k+2) \gamma}{s \mu+(k+1) \gamma} \\
& =\rho_{k+s+1}-\frac{\lambda-s \mu}{s \mu+(k+1) \gamma}\left(s ! \mu^{s} \sum_{i=0}^{s} \phi_{i}+\sum_{i=s+1}^{k+s} \rho_{i}\right)+\frac{\gamma}{s \mu+(k+1) \gamma} \sum_{i=s+1}^{k+s}(i-s) \rho_{i} .
\end{aligned}
$$

From Equations (A.8) and (A.11), we next deduce that

$$
\begin{aligned}
& U_{k+1}\left(s ! \mu^{s} \sum_{i=0}^{s} \phi_{i}+\sum_{i=s+1}^{k+s+2} \rho_{i}\right)\left(s ! \mu^{s} \sum_{i=0}^{s} \phi_{i}+\sum_{i=s+1}^{k+s+1} \rho_{i}\right) \frac{1}{\rho_{k+s+1}} \cdot \frac{s \mu+(k+2) \gamma}{s \mu+(k+1) \gamma} \\
&=U_{k}\left(s ! \mu^{s} \sum_{i=0}^{s} \phi_{i}+\sum_{i=s+1}^{k+s+1} \rho_{i}\right)\left(s ! \mu^{s} \sum_{i=0}^{s} \phi_{i}+\sum_{i=s+1}^{k+s} \rho_{i}\right) \frac{1}{\rho_{k+s}}
\end{aligned}
$$

Finally, simplifying Equation (A.12) and again observing that $\frac{\rho_{k+s+1}}{\rho_{k+s}}=\frac{\lambda}{s \mu+(k+1) \gamma}$, we get for all $k \geq 0$,

$$
U_{k+1}=\frac{s ! \mu^{s} \sum_{i=0}^{s} \phi_{i}+\sum_{i=s+1}^{k+s} \rho_{i}}{s ! \mu^{s} \sum_{i=0}^{s} \phi_{i}+\sum_{i=s+1}^{k+s+2} \rho_{i}} \cdot \frac{\lambda}{s \mu+(k+2) \gamma} \cdot U_{k},
$$

which completes the proof of the property. 


\section{B Numerical illustrations}

In this appendix, we present numerical examples to illustrate the convexity results. We compute the probability of being served as a function of the queue capacity for several systems chosen so as to cover a broad range of parameters values. Systems parameters are presented in Section 5 . 


\begin{tabular}{|c|c|c|c|c|c|c|c|c|c|}
\hline & \multicolumn{3}{|c|}{$\lambda=1.8$} & \multicolumn{3}{|c|}{$\lambda=8$} & \multicolumn{3}{|c|}{$\lambda=60$} \\
\hline$k$ & $s=1$ & $s=2$ & $s=3$ & $s=5$ & $s=10$ & $s=15$ & $s=50$ & $s=70$ & $s=100$ \\
\hline 0 & 35.7143 & 63.3484 & 81.9733 & 52.0992 & 87.8339 & 99.0899 & 78.3881 & 97.6256 & 99.9999 \\
\hline 1 & 44.3548 & 73.3209 & 89.1589 & 56.3701 & 90.9867 & 99.5033 & 79.3985 & 98.0027 & 100.0000 \\
\hline 2 & 47.5087 & 77.5281 & 92.0093 & 58.5389 & 92.9632 & 99.7085 & 80.1610 & 98.3100 & 100.0000 \\
\hline 3 & 48.8651 & 79.3467 & 93.0916 & 59.7405 & 94.2080 & 99.8076 & 80.7453 & 98.5601 & 100.0000 \\
\hline 4 & 49.4796 & 80.1024 & 93.4736 & 60.4509 & 94.9854 & 99.8542 & 81.1989 & 98.7634 & 100.0000 \\
\hline 5 & 49.7536 & 80.3947 & 93.5977 & 60.8924 & 95.4627 & 99.8755 & 81.5551 & 98.9283 & 100.0000 \\
\hline 6 & 49.8691 & 80.4986 & 93.6349 & 61.1773 & 95.7490 & 99.8850 & 81.8377 & 99.0615 & 100.0000 \\
\hline 7 & 49.9140 & 80.5324 & 93.6452 & 61.3664 & 95.9160 & 99.8890 & 82.0639 & 99.1688 & 100.0000 \\
\hline 8 & 49.9299 & 80.5425 & 93.6478 & 61.4942 & 96.0106 & 99.8908 & 82.2466 & 99.2548 & 100.0000 \\
\hline 9 & 49.9352 & 80.5454 & 93.6485 & 61.5815 & 96.0625 & 99.8915 & 82.3952 & 99.3235 & 100.0000 \\
\hline 10 & 49.9367 & 80.5461 & 93.6486 & 61.6411 & 96.0901 & 99.8918 & 82.5169 & 99.3780 & 100.0000 \\
\hline 11 & 49.9372 & 80.5463 & 93.6487 & 61.6815 & 96.1044 & 99.8919 & 82.6174 & 99.4211 & 100.0000 \\
\hline 12 & 49.9373 & 80.5463 & 93.6487 & 61.7086 & 96.1115 & 99.8919 & 82.7008 & 99.4550 & 100.0000 \\
\hline 13 & 49.9373 & 80.5463 & 93.6487 & 61.7264 & 96.1149 & 99.8919 & 82.7704 & 99.4815 & 100.0000 \\
\hline 14 & 49.9373 & 80.5463 & 93.6487 & 61.7379 & 96.1165 & 99.8919 & 82.8289 & 99.5021 & 100.0000 \\
\hline 15 & 49.9373 & 80.5463 & 93.6487 & 61.7450 & 96.1173 & 99.8919 & 82.8782 & 99.5180 & 100.0000 \\
\hline 16 & 49.9373 & 80.5463 & 93.6487 & 61.7494 & 96.1176 & 99.8919 & 82.9200 & 99.5302 & 100.0000 \\
\hline 17 & 49.9373 & 80.5463 & 93.6487 & 61.7519 & 96.1178 & 99.8919 & 82.9556 & 99.5395 & 100.0000 \\
\hline 18 & 49.9373 & 80.5463 & 93.6487 & 61.7534 & 96.1178 & 99.8919 & 82.9860 & 99.5466 & 100.0000 \\
\hline 19 & 49.9373 & 80.5463 & 93.6487 & 61.7542 & 96.1178 & 99.8919 & 83.0121 & 99.5519 & 100.0000 \\
\hline 20 & 49.9373 & 80.5463 & 93.6487 & 61.7546 & 96.1179 & 99.8919 & 83.0345 & 99.5559 & 100.0000 \\
\hline 21 & 49.9373 & 80.5463 & 93.6487 & 61.7548 & 96.1179 & 99.8919 & 83.0539 & 99.5589 & 100.0000 \\
\hline 22 & 49.9373 & 80.5463 & 93.6487 & 61.7549 & 96.1179 & 99.8919 & 83.0706 & 99.5611 & 100.0000 \\
\hline 23 & 49.9373 & 80.5463 & 93.6487 & 61.7550 & 96.1179 & 99.8919 & 83.0851 & 99.5627 & 100.0000 \\
\hline 24 & 49.9373 & 80.5463 & 93.6487 & 61.7550 & 96.1179 & 99.8919 & 83.0977 & 99.5639 & 100.0000 \\
\hline 25 & 49.9373 & 80.5463 & 93.6487 & 61.7550 & 96.1179 & 99.8919 & 83.1087 & 99.5648 & 100.0000 \\
\hline 26 & 49.9373 & 80.5463 & 93.6487 & 61.7550 & 96.1179 & 99.8919 & 83.1182 & 99.5654 & 100.0000 \\
\hline 27 & 49.9373 & 80.5463 & 93.6487 & 61.7550 & 96.1179 & 99.8919 & 83.1265 & 99.5658 & 100.0000 \\
\hline 28 & 49.9373 & 80.5463 & 93.6487 & 61.7550 & 96.1179 & 99.8919 & 83.1337 & 99.5662 & 100.0000 \\
\hline 29 & 49.9373 & 80.5463 & 93.6487 & 61.7550 & 96.1179 & 99.8919 & 83.1399 & 99.5664 & 100.0000 \\
\hline 30 & 49.9373 & 80.5463 & 93.6487 & 61.7550 & 96.1179 & 99.8919 & 83.1454 & 99.5665 & 100.0000 \\
\hline
\end{tabular}

Table 1: Values of $Q_{k}$ (in \%) for $\gamma=0.5$ 


\begin{tabular}{|c|c|c|c|c|c|c|c|c|c|}
\hline & \multicolumn{3}{|c|}{$\lambda=1.8$} & \multicolumn{3}{|c|}{$\lambda=8$} & \multicolumn{3}{|c|}{$\lambda=60$} \\
\hline$k$ & $s=1$ & $s=2$ & $s=3$ & $s=5$ & $s=10$ & $s=15$ & $s=50$ & $s=70$ & $s=100$ \\
\hline 0 & 35.7143 & 63.3484 & 81.9733 & 52.0992 & 87.8339 & 99.0899 & 78.3881 & 97.6256 & 99.9999 \\
\hline 1 & 42.9864 & 71.9585 & 88.3281 & 56.1529 & 90.8551 & 99.4904 & 79.3906 & 98.0001 & 100.0000 \\
\hline 2 & 45.2522 & 74.8962 & 90.3886 & 58.1089 & 92.6103 & 99.6776 & 80.1380 & 98.3012 & 100.0000 \\
\hline 3 & 46.0253 & 75.8487 & 90.9857 & 59.1433 & 93.6035 & 99.7606 & 80.7026 & 98.5414 & 100.0000 \\
\hline 4 & 46.2760 & 76.1248 & 91.1378 & 59.7245 & 94.1442 & 99.7954 & 81.1343 & 98.7317 & 100.0000 \\
\hline 5 & 46.3486 & 76.1951 & 91.1719 & 60.0621 & 94.4250 & 99.8094 & 81.4682 & 98.8810 & 100.0000 \\
\hline 6 & 46.3671 & 76.2108 & 91.1787 & 60.2603 & 94.5635 & 99.8147 & 81.7291 & 98.9972 & 100.0000 \\
\hline 7 & 46.3713 & 76.2140 & 91.1799 & 60.3754 & 94.6283 & 99.8166 & 81.9351 & 99.0867 & 100.0000 \\
\hline 8 & 46.3721 & 76.2146 & 91.1801 & 60.4406 & 94.6570 & 99.8173 & 82.0990 & 99.1549 & 100.0000 \\
\hline 9 & 46.3723 & 76.2147 & 91.1802 & 60.4761 & 94.6690 & 99.8175 & 82.2305 & 99.2063 & 100.0000 \\
\hline 10 & 46.3723 & 76.2147 & 91.1802 & 60.4945 & 94.6739 & 99.8176 & 82.3367 & 99.2447 & 100.0000 \\
\hline 11 & 46.3723 & 76.2147 & 91.1802 & 60.5036 & 94.6757 & 99.8176 & 82.4229 & 99.2730 & 100.0000 \\
\hline 12 & 46.3723 & 76.2147 & 91.1802 & 60.5078 & 94.6764 & 99.8176 & 82.4932 & 99.2937 & 100.0000 \\
\hline 13 & 46.3723 & 76.2147 & 91.1802 & 60.5097 & 94.6766 & 99.8176 & 82.5508 & 99.3086 & 100.0000 \\
\hline 14 & 46.3723 & 76.2147 & 91.1802 & 60.5105 & 94.6767 & 99.8176 & 82.5980 & 99.3192 & 100.0000 \\
\hline 15 & 46.3723 & 76.2147 & 91.1802 & 60.5108 & 94.6767 & 99.8176 & 82.6368 & 99.3267 & 100.0000 \\
\hline 16 & 46.3723 & 76.2147 & 91.1802 & 60.5109 & 94.6767 & 99.8176 & 82.6687 & 99.3320 & 100.0000 \\
\hline 17 & 46.3723 & 76.2147 & 91.1802 & 60.5110 & 94.6767 & 99.8176 & 82.6949 & 99.3356 & 100.0000 \\
\hline 18 & 46.3723 & 76.2147 & 91.1802 & 60.5110 & 94.6767 & 99.8176 & 82.7163 & 99.3380 & 100.0000 \\
\hline 19 & 46.3723 & 76.2147 & 91.1802 & 60.5110 & 94.6767 & 99.8176 & 82.7338 & 99.3397 & 100.0000 \\
\hline 20 & 46.3723 & 76.2147 & 91.1802 & 60.5110 & 94.6767 & 99.8176 & 82.7481 & 99.3408 & 100.0000 \\
\hline 21 & 46.3723 & 76.2147 & 91.1802 & 60.5110 & 94.6767 & 99.8176 & 82.7596 & 99.3415 & 100.0000 \\
\hline 22 & 46.3723 & 76.2147 & 91.1802 & 60.5110 & 94.6767 & 99.8176 & 82.7688 & 99.3420 & 100.0000 \\
\hline 23 & 46.3723 & 76.2147 & 91.1802 & 60.5110 & 94.6767 & 99.8176 & 82.7762 & 99.3423 & 100.0000 \\
\hline 24 & 46.3723 & 76.2147 & 91.1802 & 60.5110 & 94.6767 & 99.8176 & 82.7821 & 99.3425 & 100.0000 \\
\hline 25 & 46.3723 & 76.2147 & 91.1802 & 60.5110 & 94.6767 & 99.8176 & 82.7867 & 99.3426 & 100.0000 \\
\hline 26 & 46.3723 & 76.2147 & 91.1802 & 60.5110 & 94.6767 & 99.8176 & 82.7902 & 99.3427 & 100.0000 \\
\hline 27 & 46.3723 & 76.2147 & 91.1802 & 60.5110 & 94.6767 & 99.8176 & 82.7930 & 99.3427 & 100.0000 \\
\hline 28 & 46.3723 & 76.2147 & 91.1802 & 60.5110 & 94.6767 & 99.8176 & 82.7951 & 99.3428 & 100.0000 \\
\hline 29 & 46.3723 & 76.2147 & 91.1802 & 60.5110 & 94.6767 & 99.8176 & 82.7966 & 99.3428 & 100.0000 \\
\hline 30 & 46.3723 & 76.2147 & 91.1802 & 60.5110 & 94.6767 & 99.8176 & 82.7978 & 99.3428 & 100.0000 \\
\hline
\end{tabular}

Table 2: Values of $Q_{k}$ (in \%) for $\gamma=1$ 


\begin{tabular}{|c|c|c|c|c|c|c|c|c|c|}
\hline & \multicolumn{3}{|c|}{$\lambda=1.8$} & \multicolumn{3}{|c|}{$\lambda=8$} & \multicolumn{3}{|c|}{$\lambda=60$} \\
\hline$k$ & $s=1$ & $s=2$ & $s=3$ & $s=5$ & $s=10$ & $s=15$ & $s=50$ & $s=70$ & $s=100$ \\
\hline 0 & 35.7143 & 63.3484 & 81.9733 & 52.0992 & 87.8339 & 99.0899 & 78.3881 & 97.6256 & 99.9999 \\
\hline 1 & 41.2371 & 70.1107 & 87.1346 & 55.7787 & 90.6222 & 99.4669 & 79.3752 & 97.9950 & 100.0000 \\
\hline 2 & 42.5412 & 71.7812 & 88.3617 & 57.3866 & 92.0354 & 99.6247 & 80.0936 & 98.2842 & 100.0000 \\
\hline 3 & 42.8391 & 72.1385 & 88.6026 & 58.1445 & 92.6993 & 99.6847 & 80.6211 & 98.5063 & 100.0000 \\
\hline 4 & 42.8970 & 72.2021 & 88.6419 & 58.5085 & 92.9858 & 99.7055 & 81.0121 & 98.6735 & 100.0000 \\
\hline 5 & 42.9064 & 72.2116 & 88.6473 & 58.6788 & 93.0991 & 99.7122 & 81.3045 & 98.7969 & 100.0000 \\
\hline 6 & 42.9077 & 72.2129 & 88.6480 & 58.7540 & 93.1400 & 99.7142 & 81.5250 & 98.8861 & 100.0000 \\
\hline 7 & 42.9079 & 72.2130 & 88.6480 & 58.7848 & 93.1537 & 99.7147 & 81.6922 & 98.9493 & 100.0000 \\
\hline 8 & 42.9079 & 72.2130 & 88.6480 & 58.7964 & 93.1578 & 99.7148 & 81.8195 & 98.9931 & 100.0000 \\
\hline 9 & 42.9079 & 72.2130 & 88.6480 & 58.8004 & 93.1590 & 99.7149 & 81.9164 & 99.0228 & 100.0000 \\
\hline 10 & 42.9079 & 72.2130 & 88.6480 & 58.8017 & 93.1594 & 99.7149 & 81.9901 & 99.0426 & 100.0000 \\
\hline 11 & 42.9079 & 72.2130 & 88.6480 & 58.8021 & 93.1594 & 99.7149 & 82.0460 & 99.0555 & 100.0000 \\
\hline 12 & 42.9079 & 72.2130 & 88.6480 & 58.8022 & 93.1595 & 99.7149 & 82.0879 & 99.0637 & 100.0000 \\
\hline 13 & 42.9079 & 72.2130 & 88.6480 & 58.8022 & 93.1595 & 99.7149 & 82.1192 & 99.0688 & 100.0000 \\
\hline 14 & 42.9079 & 72.2130 & 88.6480 & 58.8023 & 93.1595 & 99.7149 & 82.1422 & 99.0720 & 100.0000 \\
\hline 15 & 42.9079 & 72.2130 & 88.6480 & 58.8023 & 93.1595 & 99.7149 & 82.1588 & 99.0739 & 100.0000 \\
\hline 16 & 42.9079 & 72.2130 & 88.6480 & 58.8023 & 93.1595 & 99.7149 & 82.1708 & 99.0750 & 100.0000 \\
\hline 17 & 42.9079 & 72.2130 & 88.6480 & 58.8023 & 93.1595 & 99.7149 & 82.1791 & 99.0756 & 100.0000 \\
\hline 18 & 42.9079 & 72.2130 & 88.6480 & 58.8023 & 93.1595 & 99.7149 & 82.1849 & 99.0760 & 100.0000 \\
\hline 19 & 42.9079 & 72.2130 & 88.6480 & 58.8023 & 93.1595 & 99.7149 & 82.1888 & 99.0762 & 100.0000 \\
\hline 20 & 42.9079 & 72.2130 & 88.6480 & 58.8023 & 93.1595 & 99.7149 & 82.1914 & 99.0763 & 100.0000 \\
\hline 21 & 42.9079 & 72.2130 & 88.6480 & 58.8023 & 93.1595 & 99.7149 & 82.1930 & 99.0763 & 100.0000 \\
\hline 22 & 42.9079 & 72.2130 & 88.6480 & 58.8023 & 93.1595 & 99.7149 & 82.1941 & 99.0764 & 100.0000 \\
\hline 23 & 42.9079 & 72.2130 & 88.6480 & 58.8023 & 93.1595 & 99.7149 & 82.1948 & 99.0764 & 100.0000 \\
\hline 24 & 42.9079 & 72.2130 & 88.6480 & 58.8023 & 93.1595 & 99.7149 & 82.1952 & 99.0764 & 100.0000 \\
\hline 25 & 42.9079 & 72.2130 & 88.6480 & 58.8023 & 93.1595 & 99.7149 & 82.1954 & 99.0764 & 100.0000 \\
\hline 26 & 42.9079 & 72.2130 & 88.6480 & 58.8023 & 93.1595 & 99.7149 & 82.1956 & 99.0764 & 100.0000 \\
\hline 27 & 42.9079 & 72.2130 & 88.6480 & 58.8023 & 93.1595 & 99.7149 & 82.1956 & 99.0764 & 100.0000 \\
\hline 28 & 42.9079 & 72.2130 & 88.6480 & 58.8023 & 93.1595 & 99.7149 & 82.1957 & 99.0764 & 100.0000 \\
\hline 29 & 42.9079 & 72.2130 & 88.6480 & 58.8023 & 93.1595 & 99.7149 & 82.1957 & 99.0764 & 100.0000 \\
\hline 30 & 42.9079 & 72.2130 & 88.6480 & 58.8023 & 93.1595 & 99.7149 & 82.1957 & 99.0764 & 100.0000 \\
\hline
\end{tabular}

Table 3: Values of $Q_{k}$ (in \%) for $\gamma=2$ 